\title{
Sponge Dosage Form
}

National Cancer Institute

\section{Source}

National Cancer Institute. Sponge Dosage Form. NCI Thesaurus. Code C47912.

A solid composed of a porous, interlacing, absorbent, usually shape retaining material, such as cellulose wood fibers or plastic polymer form. 